\title{
Somatic mosaicism for a heterozygous deletion of the survival motor neuron (SMN1) gene
}

\author{
Thomas Eggermann ${ }^{*},{ }^{1}$, Klaus Zerres ${ }^{1}$, Dirk Anhuf ${ }^{1}$, Dieter Kotzot ${ }^{2}$, Christine Fauth $^{3}$ and \\ Sabine Rudnik-Schöneborn ${ }^{1}$
}

${ }^{1}$ Institute of Human Genetics, University of Technology, Aachen, Germany; ${ }^{2}$ Institute of Human Genetics, Innsbruck, Austria; ${ }^{3}$ Institute of Human Genetics, Technical University München, Neuherberg, Germany

Infantile spinal muscular atrophy (SMA) is a common autosomal recessive disease with a high demand for carrier testing. The disease is caused by homozygous deletions of the survival motor neuron (SMN)1 gene on chromosome $5 q 13$ in more than $90 \%$ of cases. Meanwhile, several reliable quantitative methods for carrier detection in the general population have been implemented with a risk of at least $5 \%$ for false negative results. Linkage analyses with chromosome 5 markers can be used for complementary information, but they are restricted to risk estimation of close relatives in affected families. Here, we present the first observation of a somatic mosaicism in an SMA carrier. Molecular genetic studies gave evidence that the SMN1 deletion of an SMA type I patient most probably arose from somatic mosaicism in the paternal grandmother. The patient's father and his two brothers were shown to be carriers of three different maternal haplotypes in 5q13. Final conclusions for genetic counselling were only possible after both linkage analysis and quantitative real-time PCR analysis of SMN1 copy numbers.

European Journal of Human Genetics (2005) 13, 309-313. doi:10.1038/sj.ejhg.5201268

Published online 8 December 2004

Keywords: spinal muscular atrophy; somatic mosaicism; SMN1 deletion

\section{Introduction}

Infantile spinal muscular atrophy (SMA) represents one of the most common autosomal recessive diseases often leading to death in early infancy. The disorder is defined by a progressive degeneration of anterior horn cells of the spinal cord, resulting in progressive symmetrical limb and trunk paralysis associated with muscular atrophy. Patients are classified into three types, SMA I-III on the basis of age of onset and clinical severity. ${ }^{1}$ The SMA-determining gene, the survival motor neuron gene (SMN) is localised in 5q11.2q13.3 within a large inverted duplication of a $500 \mathrm{~kb}$ element. $^{2}$ The SMN gene exists in two highly homologous copies, SMN1 (the telomeric copy) and SMN2 (the

*Correspondence: $\mathrm{Dr}$ T Eggermann, Institute of Human Genetics, University Hospital, University of Technology, Aachen, Pauwelsstr. 30, D-52074 Aachen, Germany. Tel.: +49 241 8088008; Fax: +49 241 8082394; E-mail: teggermann@ukaachen.de

Received 18 February 2004; revised 8 July 2004; accepted 13 July 2004 centromeric copy). The coding region of the SMN1 gene differs from that of the SMN2 gene by only a single nucleotide in exon 7. Both $S M N$ copies are expressed, but the nucleotide difference in SMN2 causes exon 7 skipping, resulting in predominantly truncated transcripts. The SMN1 gene is homozygously deleted in more than $90 \%$ of SMA patients irrespective of clinical severity.

SMN1 dosage analysis is used to identify patients with compound mutations and for carrier detection. It is routinely performed by quantitative analysis, now mainly based on real-time PCR technologies, ${ }^{3-6}$ or by linkage analysis in SMA families. However, the presence of two SMN1 copies on the unaffected chromosome in $3-4 \%$ of normal individuals $s^{3,5,7}$ as well as the existence of subtle mutations reduces the sensitivity of quantitative approaches, therefore allowing an accurate identification of SMN1 deletion carriers in the normal population in only $\sim 95 \%$ of cases. $^{6}$ Linkage analysis in first-degree relatives circumvents this risk of a false negative result obtained by 
quantitative analysis of $S M N$ copies in a carrier of two SMN1 copies on the same chromosome. In contrast, linkage analysis rather gives false positive results, as possible new mutations in a patient (reported in $1 \%$ of parental germ cells ${ }^{8}$ ) cannot be detected. However, indirect genotype analysis is often limited if DNA samples from key family members are not available.

Here, we present molecular genetic results of a family where the SMN1 deletion of an SMA I patient most likely arose from a somatic mosaicism in the paternal grandmother. The patient's father and his two brothers were shown to be carriers of three different maternal haplotypes in 5q13. The grandmother's lymphocytes showed a reduction of genetic markers in the $S M N$ region and a reduced dosage of SMN1 in the quantitative analysis of SMN copy numbers.

\section{Materials and methods \\ Family}

The index patient (III.1) was affected by SMA type I. The clinical diagnosis was confirmed by molecular genetic detection of a homozygous SMN1 deletion. The patient's paternal uncles requested genetic testing to estimate their carrier risk. Under the assumption that the patient's father is a carrier, as is the case in about $99 \%$ of parents, his brothers had a statistical carrier risk of $50 \%$. The paternal de novo mutation rate can be estimated to be $\mu=1.6 \times 10^{-4}$ based on preliminary calculations. ${ }^{3}$ A linkage analysis was offered first to clarify the carrier status, since the probability of a false negative result in the quantitative analysis due to more than one SMN1 gene copy on the wild-type chromosome was considered to be higher than the likelihood of a new mutation.

\section{DNA studies}

Genomic DNA was isolated from peripheral lymphocytes by a simple salting-out procedure. ${ }^{13}$ Testing for the homozygous deletion of SMN1 in the index patient was performed by conventional PCR of exons 7 and 8 followed by restriction digest with HinfIII and DdeI as described previously. ${ }^{10}$

The whole family was genotyped for the microsatellite markers D5S681, D5S629, Ag1, C212, D5S557 and D5S610, which are localised in or in close neighbourhood to the SMA region. ${ }^{11}$

SMN1 copy numbers in the nonaffected family members were determined using a real-time PCR approach based on TaqMan technology using an MGB probe specific for exon 7 of SMN1 as described recently. ${ }^{6}$ As reference locus for quantification, we used a probe complementary to exon 3 of the factor VIII gene. ${ }^{6}$ For each family member, the results were confirmed in more than three independent real-time PCR runs.

\section{Results}

Linkage analysis in the family was carried out with molecular markers within and flanking the SMA region on $5 q 13$ (Figure 1) to determine the carrier status of II.3 and II.4. While the microsatellites distal and proximal of the SMA region showed two maternal haplotypes in the carrier parent and his two brothers, typing of the multicopy markers Ag1 and C212 indicated the inheritance of three different grandmaternal haplotypes (Figure 2). While brother II.3 inherited the nonaffected grandmaternal allele, marker analysis allowed to define the affected haplotype in the patient's father II.2. Interestingly, the younger brother II.4 showed full peaks of further alleles of the analysed multicopy markers, which could also be detected, but in smaller size, in the grandmaternal DNA (Figure 2).

Owing to the inconclusive results by linkage analysis, which was best explained by a somatic mosaic in the grandmother but could also be due to an allelic loss, that is, a deletion in II.2, real-time PCR of SMN1 copy number was performed in the family (Table 1 ). With the exception of individual I.2, all measured copy numbers were in the range defined by Anhuf et al; ${ }^{6}$ hence, the carrier classification corresponded well with the linkage results. The patient's father II. 2 showed an amplification pattern consistent with one SMN1 copy as expected, the patient's two uncles (II.3, II.4) were both determined to be noncarriers of the heterozygous SMN1 deletion. The grandmother showed an amplification pattern corresponding to 1.34 SMN1 copies, an intermediate value between the clearcut ranges for one (0.6-1.2) and two (1.56-2.2) SMN 1 copies. $^{6}$

These data indicated the presence of two different cell lines in the peripheral lymphocytes of the patient's grandmother with one clone containing two unaffected chromosomes 5, defined by the haplotypes 100/112-214/220 and 108/112-230/234 (multicopy alleles for Ag1-C212). The other cell line also carried the unaffected chromosome 108/112-230/234, while the second chromosome carried a deletion within the SMA region resulting in the deletion of the SMN1 copy and in reduction of multicopy marker alleles Ag1 and C212. Consequently, the 'new' mutant haplotype 112-214 originated. We conclude that the mosaicism that was detectable in peripheral lymphocytes most likely was also present in the germ cells, as the grandmother transmitted three different haplotypes to her three sons. Regarding the grandpaternal haplotypes, the three brothers (II.2, II.3, II.4) were haploidentical (108/ 110-228 for markers Ag1-C212). Altogether, both brothers II.3 and II.4 were regarded as noncarriers despite the fact that they inherited different maternal chromosomes.

\section{Discussion}

Our findings point towards somatic mosaicism for a deletion in the SMA region resulting in partial loss of the 


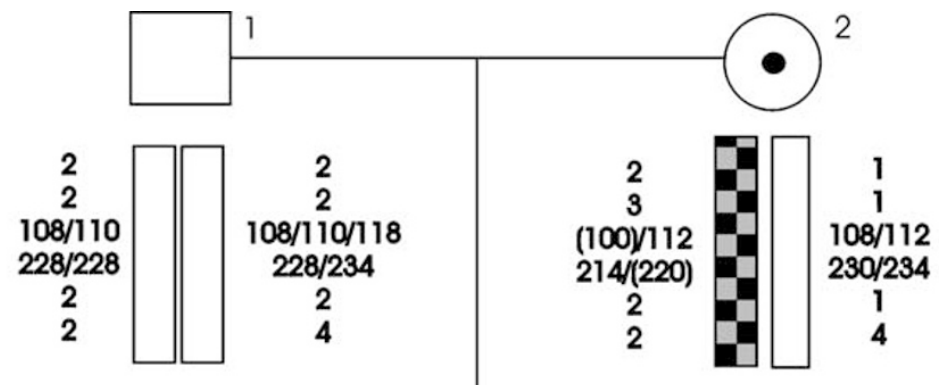

II
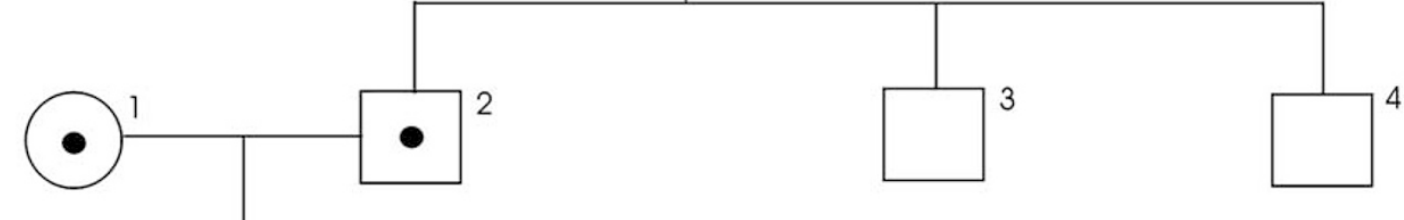

$\begin{array}{cc}\text { D5S681 } & 2 \\ \text { D5S629 } & 1 \\ \text { Ag 1 } & 110 \\ \text { C212 } & 216 \\ \text { D5S557 } & 2 \\ \text { D5S610 } & 1\end{array} \quad\left[\begin{array}{c}1 \\ 1 \\ 112 \\ 232 \\ 2 \\ 3\end{array}\right.$
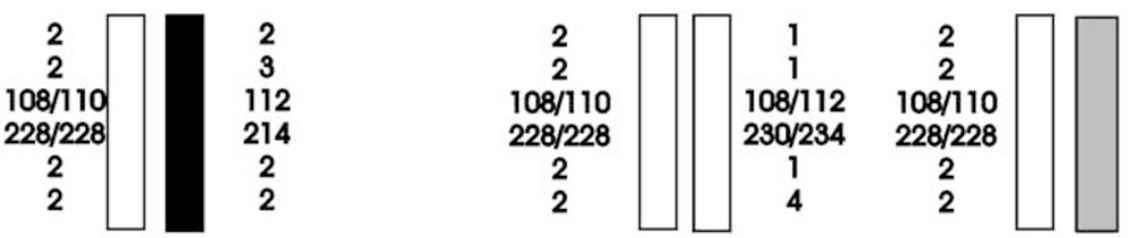

III

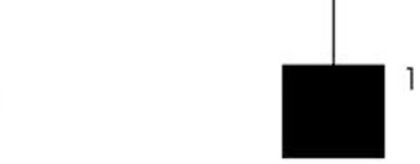

\begin{tabular}{cc|c} 
D5S681 & 2 \\
D5S629 & 1 & 2 \\
Ag1 & 110 \\
C212 & 216 \\
D5S557 & 2 \\
D5S610 & 1 & 112 \\
214 \\
\end{tabular}

Figure 1 Genotype analysis of three generations of the presented type I SMA family. Markers used are shown on the left. Black bars represent the paternal and maternal mutant chromosome 5. The grandmaternal affected chromosome 5 is shaded in grey and black. The grandmaternal Ag1 and C212 alleles written in parentheses illustrate that these signals are weaker in comparison to the other alleles (see Figure 2). Individuals identified as SMN1 deletion carriers by quantitative real-time PCR are marked by dots.

SMN1 gene at least in lymphocytes and germ cells of the patient's grandmother. While a de novo deletion in the carrier father cannot be excluded as a principle explanation, since oocytes of his mother were and are not available for investigation, the results of both linkage studies and quantitative analysis are in accordance with our hypothesis. Only rare family constellations will provide evidence of such a somatic mosaicism, since the probability of detecting ambiguous results increases with the number of persons at risk and the number of preceding generations available for analysis.

Previously, new SMN1 gene deletions were interpreted as germ cell mutations mainly attributed to unequal recombinations during paternal meiosis. ${ }^{8}$ Meanwhile, it was shown that new deletions can also occur in maternal germ cells. ${ }^{12}$ In addition, germline mosaicism has been observed in a family with three affected children, whose mother harboured a second mutation event on her mutant SMA 

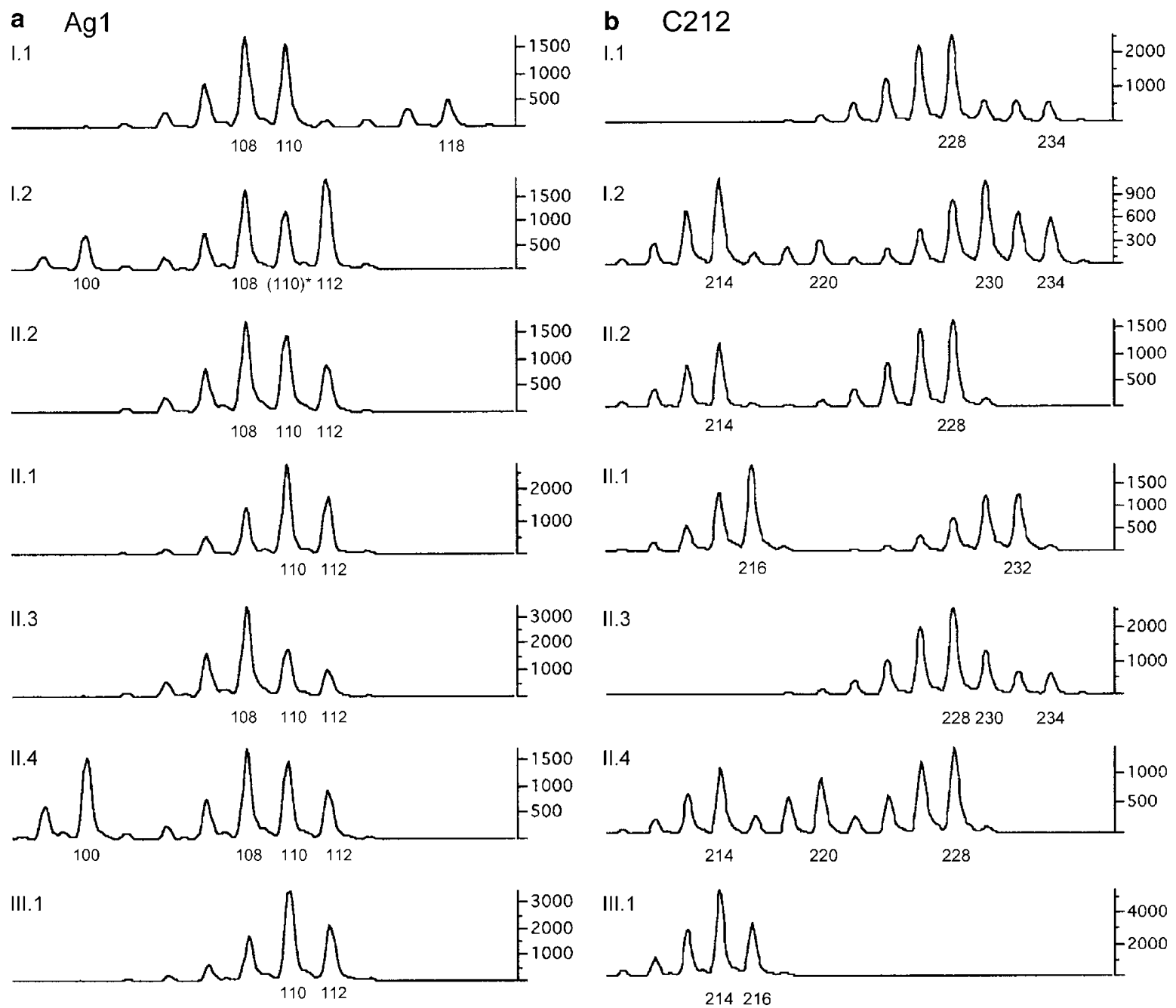

Figure 2 Inheritance of the multicopy markers (a) Ag1 and (b) C212 alleles in the SMA family presented here. (a) While proband II. 3 had inherited the alleles 108/112 from his mother, the two sons II.2 and II.4 shared the allele 112, which is linked to the SMN1 deletion. However, individual II.4 carried an additional allele 100, which was detectable in his mother's DNA but in none of his brothers samples. (110)*: The peak corresponding to allele 110 in II.2 was interpreted as an unspecific amplification product; however, the presence of mosaicism for this allele cannot be ruled out. (b) The same pattern of inheritance was observed by C212 typing: the two brothers II. 2 and II.4 shared the grandmaternal allele 214, while II.4 carried an additional allele 220 which was not seen in his brothers. Allele sizes determined by the Genescan analysis software (Appliedbiosystems, Darmstadt, Germany) are given below each electropherogram; to facilitate the allele calling in the text and Figure 1, allele sizes were rounded up.

Table 1 Results of real-time PCR quantification of the SMN1 copy number in the presented SMA family

\begin{tabular}{lcc}
\hline Proband & $\begin{array}{c}\text { SMN1 mean } \\
\text { copy number }\end{array}$ & $\begin{array}{c}\text { SMN1 mean } \\
\text { measured copy } \\
\text { number (range) }\end{array}$ \\
\hline I.1 & 2 & $1.82(1.60-2.0)$ \\
I.2 & $1-2$ & $1.34(1.20-1.48)$ \\
II.1 & 1 & $0.94(0.70-1.00)$ \\
II.2 & 1 & $1.00(0.60-1.12)$ \\
II.3 & 2 & $2.14(1.60-2.20)$ \\
II.4 & 2 & $1.96(1.72-2.04)$ \\
\hline
\end{tabular}

Proband numbers correspond to the pedigree (Figure 1). chromosome. ${ }^{9}$ The carrier mother transmitted a large deletion (null allele) including SMN1 and SMN2 to her children who died of SMA type I. In addition, she had a cell line with the mutant maternal chromosome 5 containing two SMN2 copies, one of which had arisen by gene conversion from SMN1. The findings were best explained by maternal germline mosaicism but somatic mosaicism was also considered. However, further genotype analysis did not yield informative results. The authors concluded that their family highlighted the inherent instability, and they postulated a high mutation rate of the SMA region. 
Ogino et $\mathrm{al}^{3}$ encountered a possible case of germline mosaicism for $S M N 1$, with a variable proportion of $S M N 1$ signals in a sperm and blood sample; however, further details were not reported.

While recurrence risk is only small in families with true de novo deletions, it is not negligible in the setting of germline mosaicism. In that case, recurrence risk is unpredictable and can be somewhere between 0 and $25 \%$, which has high implications for genetic counselling and prenatal diagnosis. Nonetheless, germline or somatic mosaicism is only rarely observed in SMA, as has to be expected for autosomal recessive conditions being in accordance with the mutation-selection equilibrium.

Our family illustrates the advantages and disadvantages of the different direct and indirect strategies performed in SMN1 deletion carrier testing: linkage analysis generally allows a more exact determination of carriership in comparison to quantitative methods but this approach failed in our family and only the quantitative real-time PCR test gave a reliable result for II.4. On the other hand, the two oocyte cell lines in the grandmother I.2 were only identified by typing of the multicopy markers Ag1 and C212. Nevertheless, the copy number determination by the new generation of quantitative tests based on real-time PCR generally allows a clearcut differentation of SMN1 copy numbers (for a review, see Anhuf et $a l^{6}$ ), except mosaic cases as impressively illustrated here.

\section{References}

1 Zerres K, Davies KE: Workshop report. Spinal muscular atrophies: recent progress and revised diagnostic criteria. Neuromusc Disord 1999; 9: $272-278$
2 Lefebvre S, Bürglen L, Reboullet $\mathrm{S}$ et al: Identification and characterisation of a spinal muscular atrophy-determining gene. Cell 1995; 80: 155-165.

3 Ogino S, Wilson RB: Genetic testing and risk assessment for spinal muscular atrophy (SMA). Hum Genet 2002; 111: 477-500.

4 Feldkötter M, Schwarzer V, Wirth R, Wienker TI, Wirth B: Quantitative analyses of SMN1 and SMN2 based on real-time LightCycler PCR: fast and highly reliable carrier testing and prediction of spinal muscular atrophy. Am J Hum Genet 2002; 70: $358-368$.

5 Cuscó I, Barceló MJ, Baiget M, Tizzano EF: Implementation of SMA carrier testing in genetic laboratories: comparison of two methods for quantifying the SMN1 gene. Hum Mut 2002; 20: $452-459$.

6 Anhuf D, Eggermann T, Rudnik-Schöneborn S, Zerres K: Determination of SMN1 and SMN2 copy number using TaqMan technology. Hum Mutat 2003; 22: 74-78.

7 Wirth B, Herz M, Wetter A et al: Quantitative analysis of survival motor neuron copies: identification of subtle mutations in patients with spinal muscular atrophy, genotype-phenotype correlation, and implications for genetic counseling. Am J Hum Genet 1999; 6: 1340-1356.

8 Wirth $\mathrm{B}$, Schmidt $\mathrm{T}$, Hahnen $\mathrm{E}$ et al: De novo rearrangements found in $2 \%$ of index patients with spinal muscular atrophy: mutational mechanisms, parental origin, mutation rate, and implications for genetic counseling. Am J Hum Genet 1997; 61: $1102-1111$.

9 Campbell L, Daniels RJ, Dubowitz V, Davies KE: Maternal mosaicism for a second mutational event in a type I spinal muscular atrophy family. Am J Hum Genet 1998; 63: 37-44.

10 Van der Steege G, Grootscholten PM, van der Vlies P et al: PCRbased test to confirm clinical diagnosis of autosomal recessive spinal muscular atrophy. Lancet 1995; 345: 985-986.

11 Scheffer H, Cobben JM, Matthijs G, Wirth B: Best practice guidelines for molecular analysis in spinal muscular atrophy. Eur J Hum Genet 2001; 8: 79-86.

12 Raclin V, Veber PS, Burglen L, Munnich A, Melki J: De novo deletions in spinal muscular atrophy: implications for genetic counselling. J Med Genet 1997; 34: 86-87.

13 Miller SA, Dykes DD, Polesky HF: A simple salting out procedure of extracting DNA from human nucleated cells. NAR 1988; 16: 1215. 